\title{
Origin of Constant Loss in Ionic Conductors
}

\author{
C. León, ${ }^{1}$ A. Rivera, ${ }^{1,2}$ A. Várez, ${ }^{3}$ J. Sanz, ${ }^{2}$ and J. Santamaria ${ }^{1}$ \\ ${ }^{1}$ GFMC, Departamento de Fisica Aplicada III, Facultad de Fisica, Universidad Complutense Madrid, \\ 28040 Madrid, Spain \\ ${ }^{2}$ Instituto de Ciencia de Materiales de Madrid (CSIC), Cantoblanco, 28049 Madrid, Spain \\ ${ }^{3}$ Departamento de Ingenieria, Escuela Politécnica Superior, Universidad Carlos III Madrid, \\ 28911 Leganés, Spain \\ K. L. Ngai \\ Naval Research Laboratory, Washington, D.C. 20375-5320
}

(Received 25 May 2000)

\begin{abstract}
We have analyzed the constant loss contribution to the ac conductivity in the frequency range $10 \mathrm{~Hz}-1 \mathrm{MHz}$ and temperatures down to $8 \mathrm{~K}$, for two $\mathrm{Li}$ ionic conductors, one crystalline $\left(\mathrm{Li}_{0.18} \mathrm{La}_{0.61} \mathrm{TiO}_{3}\right)$ and the other glassy $\left(61 \mathrm{SiO}_{2} \cdot 35 \mathrm{Li}_{2} \mathrm{O} \cdot 3 \mathrm{Al}_{2} \mathrm{O}_{3} \cdot \mathrm{P}_{2} \mathrm{O}_{5}\right)$. As temperature is increased a crossover is observed from a nearly constant loss to a fractional power law frequency dependence of the ac conductivity. At any fixed frequency $\omega$, this crossover occurs at a temperature $T$ such that $\omega \approx \nu_{0} \exp \left(-E_{m} / k_{B} T\right)$, where $\nu_{0}$ is the attempt frequency and $E_{m}$ is identified with the barrier for $\mathrm{Li}^{+}$ions to leave their wells.
\end{abstract}

DOI: 10.1103/PhysRevLett.86.1279

PACS numbers: 66.30.Hs, 66.30.Dn

Much effort has been devoted during the last few decades to understand the dynamics of ionic transport in ionically conducting materials. In spite of the advances made, there is still no general agreement on interpretation of the experimental data [1-11]. Most research activity in this field has focused on the origin and properties of the long-range ion motion, and electrical relaxation is the most commonly used experimental tool to access the ion dynamics. The frequency dependence of the ionic conductivity can be usually well described by using Jonscher's expression [12]

$$
\sigma^{*}(\omega)=\sigma_{0}\left[1+\left(j \omega / \omega_{p}\right)^{n}\right]
$$

where $\sigma_{0}$ is the de conductivity, $\omega_{p}$ is a characteristic relaxation frequency, and $n$ is a fractional exponent.

Both $\sigma_{0}$ and $\omega_{p}$ are thermally activated with about the same activation energy, indicating that the dispersive conductivity, $\sigma^{*}(\omega)$, originates from migration of ions. However, there is another ubiquitous contribution to dispersive conductivity that has received much less attention so far. This contribution consists of a nearly frequency independent dielectric loss, $\varepsilon^{\prime \prime}(\omega) \approx A$, which corresponds to an almost linear frequency dependent term of the form $\sigma^{\prime}(\omega)=\omega \varepsilon^{\prime \prime}(\omega) \approx A \omega$ in the real part of the complex conductivity. At sufficiently low temperature or high frequencies, the $A \omega$ term dominates over the power law dependence of exponent $n$. The existence of this nearly constant loss (NCL) was suggested more than 20 years ago and subsequently verified $[13,14]$. Since then, few investigations of its properties have been made [15-17] and low temperature data with its dominant contribution are still scarce.

Although, ultimately, mobile ions seem to be responsible also for the NCL, the experimental facts including its dependence on temperature and the effect of mixed alkalis point to a different origin than ionic hopping [18]. Experimentally, $A$ is not thermally activated and has temperature dependence much milder than $\sigma_{0}$ or $\omega_{p}$ [18-20]. Partial replacement by alkali ions of a different kind has the effect of reducing the NCL $[3,18,21,22]$, but the reduction in $A$ is much smaller than the decrease in $\sigma_{0}$ due to the well-known mixed alkali effect [3]. From these facts, it has been very recently proposed [18] that local vibrational relaxation reflected in the mean-square displacement of ions could be the origin of the constant loss in ionic conductors. At the shortest time scale ions are vibrating in their potential wells of height $E_{m}$, with a frequency $\nu_{0}$ usually termed attempt frequency. A microscopic residence time is defined, $\tau_{m}=\nu_{0}^{-1} \exp \left(E_{m} / k_{B} T\right)$, such that, statistically, for times $t<\tau_{m}$ ions are vibrating within their sites and for $t>\tau_{m}$ they abandon the well. This microscopic time can be obtained from the temperature dependence of the dispersive ac conductivity or from the low temperature side of $1 / T_{1}$ NMR measurements $[23,24]$.

In this Letter we investigate the vibrational origin of the NCL by electrical conductivity measurements in the audio frequency range at low temperatures. We have performed complex admittance measurements at low temperatures of the crystalline fast ionic conductor $\mathrm{Li}_{3 x} \mathrm{La}_{2 / 3-x} \mathrm{TiO}_{3}$. This ionically conducting material is a model system for this purpose since it shows a high ionic conductivity [25-28], and the highest value ever determined of the constant loss term [18]. We find that the NCL dominates the $\sigma^{\prime}(\omega)$ at time scales $t<\tau_{m}$ and observe a crossover of $\sigma^{\prime}(\omega)$ to the sublinear frequency dependence of Eq. (1) for $t>\tau_{m}$. This finding provides strong evidence for a vibrational origin of the constant loss. 
Figure 1 shows conductivity vs temperature plots at different fixed frequencies for $\mathrm{Li}_{0.18} \mathrm{La}_{0.61} \mathrm{TiO}_{3}$. At the highest temperatures, experimental data taken at different frequencies tend to collapse in a single curve, corresponding to the temperature dependent dc ionic conductivity. When temperature is lowered in the range $225-275 \mathrm{~K}$, the additional contribution to the conductivity due to the
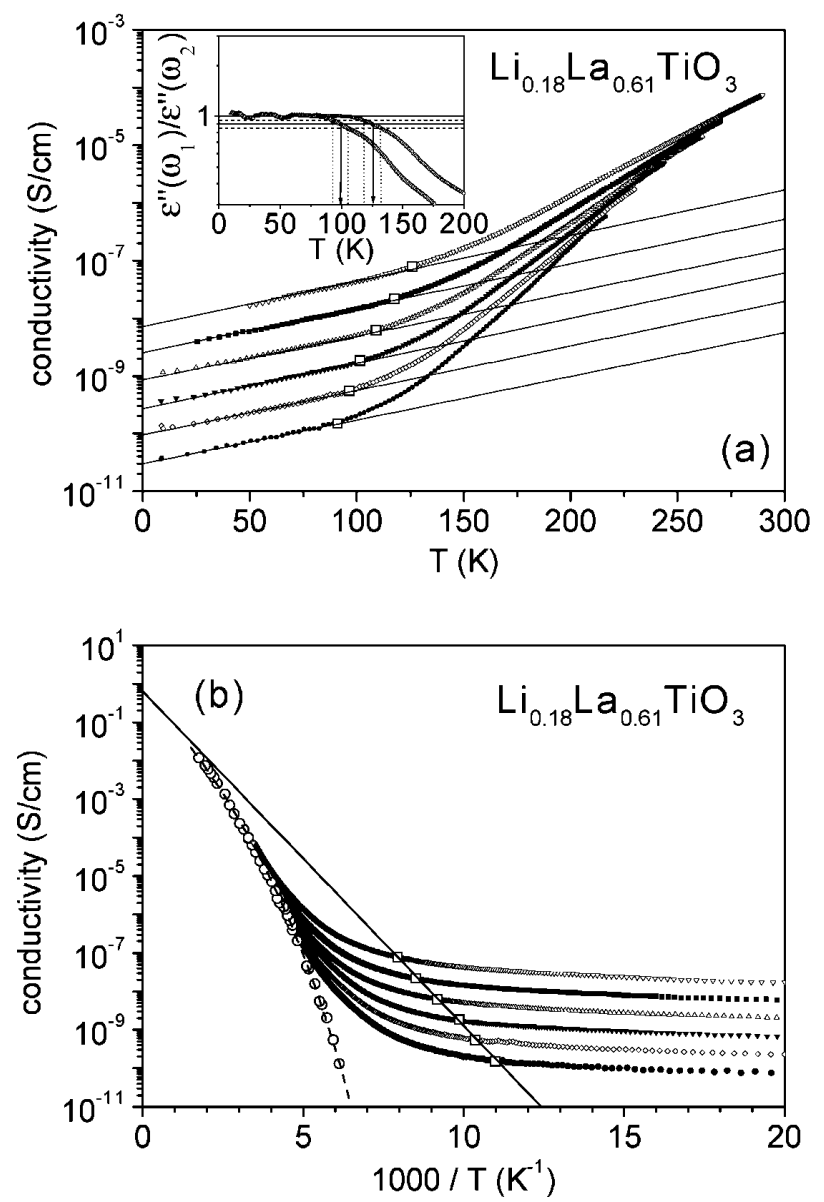

FIG. 1. Conductivity vs temperature plots for $\mathrm{Li}_{0.18} \mathrm{La}_{0.61} \mathrm{TiO}_{3}$. Conductivity data are shown for different frequencies $(300 \mathrm{~Hz}$, $1 \mathrm{kHz}, 3 \mathrm{kHz}, 10 \mathrm{kHz}, 30 \mathrm{kHz}$, and $100 \mathrm{kHz}$, from bottom to top) in a linear temperature scale (a) and in an Arrhenius plot (b). Open square symbols in both plots represent the crossover between the linear frequency dependent conductivity (constant loss regime) and the power law regime of Eq. (1) which is due to ionic hopping. Solid lines in (a) are fits to an exponential temperature dependence of conductivity data in the constant loss regime. Open circles in (b) are dc conductivity data, and the solid line is a fit to an Arrhenius law for the crossover temperature dependence. The inset in (a) shows the criterion used to obtain the crossover temperature at each frequency. The ratio of the permittivity values at two different frequencies, $\omega_{1}$ and $\omega_{2}$, is equal to 1 at low temperatures, where constant loss is the dominant contribution, but starts to depart from 1 when temperature is increased and ion hopping contributes to ac conductivity. Displayed data are for $\omega_{1}=3 \mathrm{kHz}(\Delta)$ and $100 \mathrm{kHz}$ (o), with $\omega_{2}=\omega_{1} / 10$. Crossover temperatures were obtained for a departure of $10 \%$ (arrows) and error bars of $\pm 7 \mathrm{~K}$ were obtained from $5 \%$ and $15 \%$ decays (dotted lines). power law dependence of the ionic ac conductivity is observed, first appearing for the highest frequencies. In this regime, ions have already jumped over the potential barriers of their wells but a stationary behavior, i.e., a dc conductivity value, for the long-range ionic transport has not yet been achieved. We have to lower temperatures down to $100-125 \mathrm{~K}$ to observe the dominant contribution of the NCL. Once this regime appears it is found to dominate conductivity curves up to the lowest temperatures. The NCL regime is characterized by the almost linear frequency dependence of ac conductivity and a weak temperature dependence. The best description of the temperature dependence of the NCL over a wide temperature range is the exponential form $\exp (B T)$. Solid lines in Fig. 1 are fits to an exponential temperature dependence of the ac conductivity at each frequency. Note that the low temperature portion of experimental conductivity data sets of Fig. 1(a) are spaced according to a linear frequency term. This linear frequency dependence provides a criterion to determine, at each frequency, a crossover temperature where the constant loss term becomes the dominant contribution to the ac conductivity. The crossover points, represented by open squares in Fig. 1(a), were obtained from the highest temperature where a linear frequency dependence was still observed within a $10 \%$ accuracy. The same ac conductivity data of Fig. 1(a) are displayed in an Arrhenius manner in Fig. 1(b). Conductivity data at the crossover temperatures and for each frequency (open squares) show a thermally activated behavior with an activation energy $E=0.17 \pm 0.03 \mathrm{eV}$. The very same value is obtained from spin-lattice and electrical conductivity relaxation [27], for the barrier for short-range ion motion, $E_{m}=0.17 \pm 0.01 \mathrm{eV}$, i.e., the height of the single ion potential well. The extrapolation to infinite temperature yields a conductivity value of about $1 \mathrm{~S} / \mathrm{cm}$. It has the same order of magnitude as the dc conductivity extrapolated to infinite temperature and the dc conductivity values of other fast ionic conductors observed to saturate at high temperatures [29-31]. dc conductivity data at high temperatures show an activation energy $E_{m}$, while a larger value, $E_{\sigma}$, is found when temperature is lowered, which is usually interpreted in terms of the additional energy required for cooperative motion of ions to overcome correlations [32]. According to the Nernst-Einstein equation, $\sigma_{0}=\left(N q^{2} d^{2} \nu_{0}\right) /\left(6 k_{B} T\right)$, the extrapolated value of about $1 \mathrm{~S} / \mathrm{cm}$ is determined by an attempt frequency, $\nu_{0}$, in the range $10^{12}-10^{13} \mathrm{~s}^{-1}$, for usual mobile ion concentrations $\left(N \approx 10^{21}-10^{22} \mathrm{~cm}^{-3}\right)$ and physical ionic jump distances $d$ of a few angstroms. The NCL is then observed at a given temperature for frequencies

$$
\omega>\tau_{m}^{-1}=\nu_{0} \exp \left(-E_{m} / k_{B} T\right),
$$

and for a given frequency $\omega$ at temperatures below $E_{m} /\left[k_{B} \ln \left(\nu_{0} / \omega\right)\right]$ that ions have not abandoned their 
own sites. The solid line in Fig. 1(b) obtained from the crossover divide the plot in two temperature regions. For all frequencies $\omega$, the right side is where the temperature falls below the crossover temperature and ions remain within their sites. The left side is the regime when enough thermal energy is available, and mobile ions start to jump over their own sites and subsequently adjacent sites, giving an additional contribution to the ac conductivity, which overshadows the linear frequency term.

Moreover, and interestingly enough, we want to emphasize that this result is at odds with the usual assumption [15-22] that the total ac conductivity in ionic conductors can be described by the augmented Jonscher's expression,

$$
\sigma^{*}(\omega)=\sigma_{0}\left[1+\left(j \omega / \omega_{p}\right)^{n}\right]+A \omega,
$$

where ac hopping ionic conductivity and the NCL contribution are considered to be additive terms. Since $\sigma_{0}$ and $\omega_{p}$ are thermally activated with the dc activation energy $E_{\sigma}$, meanwhile $A$ shows a much milder temperature dependence; it can be shown from Eq. (3) that the crossover between the two terms should be thermally activated with the energy $E_{\sigma}$. This is readily obtained by equating the NCL term to the real part of the ac conductivity given by the Jonscher's expression at the crossover frequency. However, experimental data show that the activation energy of the crossover is close to $E_{m}$ and much lower than $E_{\sigma}$. This finding suggests that although Eq. (3) may phenomenologically describe the frequency behavior at a given temperature, it does not account for the temperature dependence of the conductivity in the presence of NCL contribution. This might reflect that a true crossover occurs from the NCL to the ion hopping conductivity relaxation which cannot be expressed as a simple sum.

We have also analyzed conductivity data after Hsieh and Jain [33] of the glassy lithium silicate of composition $61 \mathrm{SiO}_{2} \cdot 35 \mathrm{Li}_{2} \mathrm{O} \cdot 3 \mathrm{Al}_{2} \mathrm{O}_{3} \cdot \mathrm{P}_{2} \mathrm{O}_{5}$, on which complex admittance measurements have been reported at temperatures low enough for the NCL to become dominant. Figure 2 shows conductivity vs temperature plots by using their data. For the frequencies of measurement they used, the dc conductivity regime is not completely reached below room temperature, but the crossover between the NCL and the start of the ionic hopping process is clearly observed in the temperature range $125-150 \mathrm{~K}$. Interestingly, as we have found for $\mathrm{Li}_{0.18} \mathrm{La}_{0.61} \mathrm{TiO}_{3}$, these authors also reported that best fits of NCL were a weak exponential temperature dependence. We have estimated the crossover temperatures from these isochronal curves in the same way as done for $\mathrm{Li}_{0.18} \mathrm{La}_{0.61} \mathrm{TiO}_{3}$ and replotted their ac conductivity data in an Arrhenius plot [see Fig. 2(b)]. Again a crossover from NCL to ion hopping ac conductivity is found at frequency or temperature defined by Eq. (2). The activation energy obtained in this case was found to be $E_{m}=0.27 \pm 0.04 \mathrm{eV}$, which is the same as the activation energy $E_{m}$ found for the ac conductivity
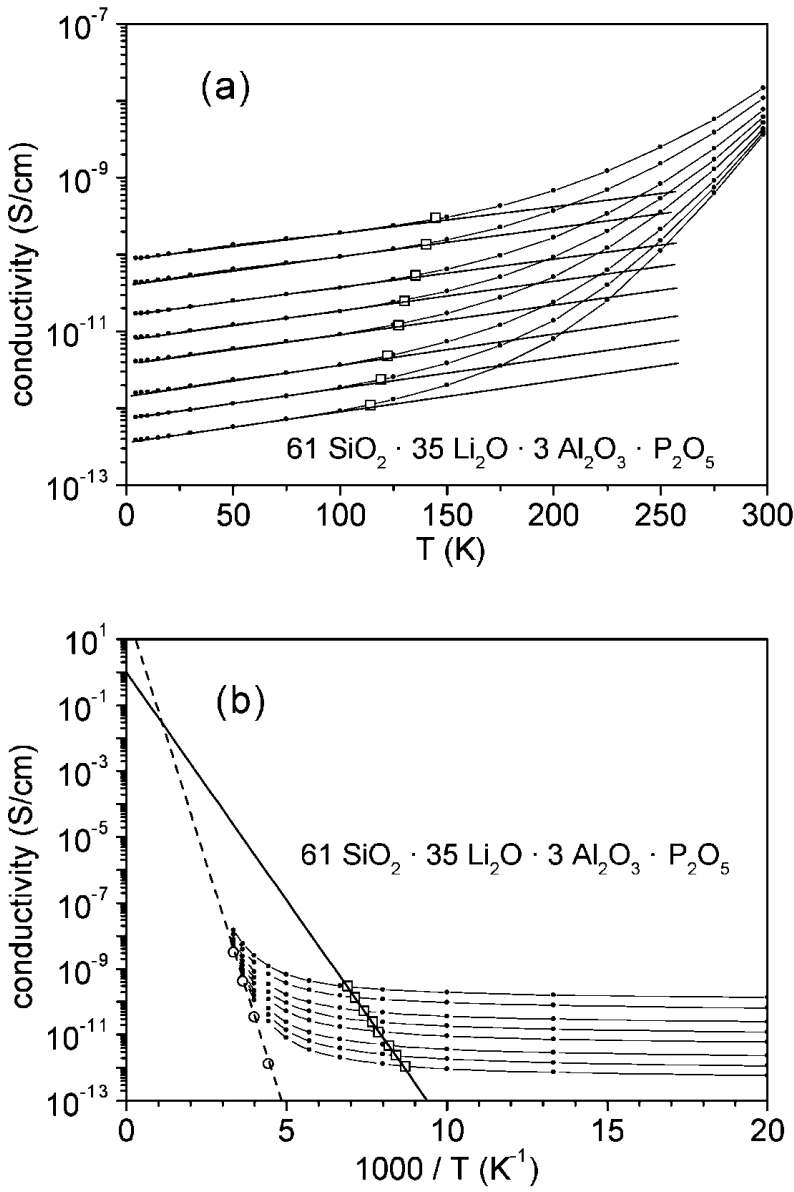

FIG. 2. Conductivity vs temperature plots for $61 \mathrm{SiO}_{2}$. $35 \mathrm{Li}_{2} \mathrm{O} \cdot 3 \mathrm{Al}_{2} \mathrm{O}_{3} \cdot \mathrm{P}_{2} \mathrm{O}_{5}$ after Hsieh and Jain [33]. Conductivity data are shown for different frequencies $(50 \mathrm{~Hz}$, $100 \mathrm{~Hz}, 200 \mathrm{~Hz}, 500 \mathrm{~Hz}, 1 \mathrm{kHz}, 2 \mathrm{kHz}, 5 \mathrm{kHz}$, and $10 \mathrm{kHz}$, from bottom to top) in a linear temperature scale (a) and in an Arrhenius plot (b). Open square symbols in both plots represent the crossover between the linear frequency dependent conductivity (constant loss regime) and the power law regime of Eq. (1) which is due to ionic hopping. Solid lines in (a) are fits to an exponential temperature dependence of conductivity data in the constant loss regime. Open circles in (b) are dc conductivity data, and the solid line is a fit to an Arrhenius law for the crossover temperature dependence.

in the power law regime at higher temperatures [33], and can be associated with the height of potential barriers of the sites where ions reside. Conductivity data values at the onset of ionic hopping extrapolate again to the expected value of about $1 \mathrm{~S} / \mathrm{cm}$ in the infinite temperature limit. Hence, in this glassy ionic conductor the NCL is the relaxational response observed when mobile ions still remain in their respective sites and ion hopping has not yet become effective. Having established this crossover, naturally the next issue is how the ions vibrating but remaining at their respective sites can generate the NCL. One possible cause is the anharmonicity of the potential well. According to recent works based on nonlinear Hamiltonian dynamics, anharmonicity alone can slow 
down immensely the relaxation of vibration [34-36]. Even the asymmetric double well potential used before to explain the NCL is a special case of anharmonicity, which has been modeled by the soft $\phi^{4}$ potential and slowed down vibration relaxation was found by computation [36]. The crossover from NCL to ion hopping (i.e., the NCL ceases when the ion diffusion starts) found from the experimental data presented here has a natural explanation in this scenario. When the time scale for the diffusion of ions by hopping becomes comparable to or shorter than the time scale for the vibrational relaxation associated with the NCL process, the NCL process can no longer take place. Therefore below the frequency or temperature defined by Eq. (2), vibrational relaxation terminates and NCL ceases to exist, and a well-defined crossover to ionic hopping ac conductivity occurs.

In summary, we have analyzed ac conductivity data of two very different ionic conductors at low temperatures and for several frequencies in the audio frequency range. We have found that the crossover from the fractional power law dependent ac conductivity term to the nearly constant loss regime characterized by a linear frequency dependent ac conductivity is thermally activated with an activation energy which is significantly lower than the dc conductivity activation energy and can be identified with the potential energy barrier that ions must overcome to abandon their sites to start hopping. This finding shows that Eq. (3) is invalid. It provides also evidence for the nearly constant loss to be due to the vibrational relaxation caused by anharmonicity.

The authors thank H. Jain for providing experimental data of the lithium silicate glass. Financial support from CICYT Grant No. MAT98-1053-C04 is also acknowledged. K.L.N. is supported by ONR.

[1] C. A. Angell, Chem. Rev. 90, 523 (1990).

[2] See the collection of papers in J. Non-Cryst. Solids 131-133 (1991); 172-174 (1994); 235-238 (1998).

[3] K. L. Ngai, J. Non-Cryst. Solids 203, 232 (1996).

[4] B. Roling, A. Happe, K. Funke, and M. D. Ingram, Phys. Rev. Lett. 78, 2160 (1997).

[5] P. Lunkenheimer, A. Pimenov, and A. Loidl, Phys. Rev. Lett. 78, 2995 (1997).

[6] C. León, M. L. Lucia, J. Santamaria, and F. SánchezQuesada, Phys. Rev. B 57, 41 (1998).

[7] K. L. Ngai and C. T. Moynihan, Bull. Mater. Res. Soc. 23, 51 (1998)
[8] D. L. Sidebottom, Phys. Rev. Lett. 82, 3653 (1999).

[9] K. L. Ngai and C. León, Phys. Rev. B 60, 9396 (1999).

[10] T. B. Schrøder and J. C. Dyre, Phys. Rev. Lett. 84, 310 (2000).

[11] A. Ghosh and A. Pan, Phys. Rev. Lett. 84, 2188 (2000).

[12] A. K. Jonscher, Dielectric Relaxation in Solids (Chelsea Dielectric Press, London, 1983).

[13] J. Wong and C. A. Angell, Glass Structure by Spectroscopy (Dekker, New York, 1976).

[14] A. Burns, G. D. Chryssikos, E. Tombari, R. H. Cole, and W. M. Risen, Phys. Chem. Glasses 30, 264 (1989).

[15] W. K. Lee, J. F. Liu, and A. S. Nowick, Phys. Rev. Lett. 67, 1559 (1991).

[16] C. Cramer, K. Funke, and T. Saatkamp, Philos. Mag. B 71, 701 (1995).

[17] K. L. Ngai, H. Jain, and O. Kanert, J. Non-Cryst. Solids 222, 383 (1997).

[18] K. L. Ngai, J. Chem. Phys. 110, 10576 (1999).

[19] C. León, M. L. Lucia, and J. Santamaria, Phys. Rev. B 55, 882 (1997)

[20] A. S. Nowick, A. V. Vaysleb, and W. Liu, Solid State Ionics 105, 121 (1998).

[21] D. L. Sidebottom, P. F. Green, and R. K. Brow, Phys. Rev. Lett. 74, 5068 (1995).

[22] H. Jain and X. Lu, J. Non-Cryst. Solids 196, 285 (1996).

[23] F. Borsa, D. R. Torgeson, S. W. Martin, and H. K. Patel, Phys. Rev. B 46, 795 (1992).

[24] K. L. Ngai, Phys. Rev. B 48, 13481 (1993).

[25] Y. Inaguma, L. Chen, M. Itoh, T. Nakamura, T. Uchida, M. Ikuta, and M. Wakihara, Solid State Commun. 86, 689 (1993).

[26] C. León, M. L. Lucia, J. Santamaria, M. A. Paris, J. Sanz, and A. Várez, Phys. Rev. B 54, 184 (1996).

[27] C. León, J. Santamaria, M. A. Paris, J. Sanz, J. Ibarra, and L. M. Torres, Phys. Rev. B 56, 5302 (1997).

[28] J. A. Alonso, J. Sanz, J. Santamaria, C. León, A. Várez, and M. T. Fernández, Angew. Chem., Int. Ed. Engl. 39, 619 (2000).

[29] J. Kincs and S.W. Martin, Phys. Rev. Lett. 76, 70 (1996).

[30] K. L. Ngai and A. K. Rizos, Phys. Rev. Lett. 76, 1296 (1996).

[31] P. Maass, M. Meyer, A. Bunde, and W. Dieterich, Phys. Rev. Lett. 77, 1528 (1996).

[32] K. L. Ngai, G. N. Greaves, and C. T. Moynihan, Phys. Rev. Lett. 80, 1018 (1998).

[33] C. H. Hsieh and H. Jain, J. Non-Cryst. Solids 203, 293 (1996)

[34] G. P. Tsironis and S. Aubry, Phys. Rev. Lett. 77, 5225 (1996).

[35] A. Bikaki, N. K. Voulgarakis, S. Aubry, and G. P. Tsironis, Phys. Rev. E 59, 1234 (1999).

[36] S. Flach and G. Mutschke, Phys. Rev. E 49, 5018 (1994). 